\title{
Creation of a Cross-Culture Collaboration Course and Examination of Its Benefits for Visiting and Host Undergraduate Students
}

\author{
Benjamin $\mathrm{Ahn}^{1} \&$ Jane A. Connor ${ }^{2}$ \\ ${ }^{1}$ Aerospace Engineering, Iowa State University, Iowa, USA \\ ${ }^{2}$ Comparative Media Studies/Writing, Massachusetts Institute of Technology, Massachusetts, USA \\ Correspondence: Benjamin Ahn, Assistant Professor, Aerospace Engineering, Iowa State University, Iowa, USA.
}

Received: November 10, 2016

Accepted: November 24, $2016 \quad$ Online Published: December 12, 2016

doi:10.5430/irhe.v2n1p33

URL: http://dx.doi.org/10.5430/irhe.v2n1p33

\begin{abstract}
The purpose of this paper is (1) to present a cross-culture collaboration (CCC) skill-training curriculum that was developed in the US for visiting Singaporean students and host US students, and (2) to examine the benefits of this curriculum for the students. The course benefits were assessed using pre- and post-class surveys and students' journal entries. This study provides insight into the value of CCC course and guides educators in teaching CCC to students in classes.
\end{abstract}

Keywords: cross-culture collaboration, intercultural communication, study abroad

\section{Introduction}

Engineers increasingly work in a diverse, interconnected, and changing world, a fact amply remarked upon by engineering educators and several national reports. According to William Wulf, former president of the National Academy of Engineering:

"[E]ngineering is now practiced in a global, holistic business context, and engineers must design under constraints that reflect that context. In the future, understanding other cultures, speaking other languages, and communicating with people from marketing and finance will be just as fundamental to the practice of engineering as physics and calculus." (Wulf 2004)

Recognizing the importance of training engineers to collaborate with people from different parts of the world, US and international universities have collaborated to offer study-abroad programs. Though these programs vary in makeup and duration, they typically include taking courses, conducting research, and participating in local projects.

As visiting students interact with host university students, we expect them to develop cross-culture collaboration (CCC) skills, that is, the ability to work with team members from various national origins and to appreciate and be sensitive to others' cultures. However, the rate and the degree to which students develop CCC skills varies. Visiting students in programs lasting only a few weeks have little opportunity to develop those skills if they do not actively interact with host university students and the programs do not actively encourage and nurture CCC skills. Even for longer-term programs, unless visiting students are given the opportunity to work with host students on a regular basis their CCC skill development will be limited.

We believe that every study-abroad program should include a course that explicitly teaches CCC skills. Such a course, taught by faculty from the host university, can provide a safe environment for visiting students to observe and discuss cultural norms and to practice communication and intercultural collaboration skills. Furthermore, opening the course to host students allows both host and visiting students to learn CCC skills from each other, so that they all become able to communicate and collaborate effectively with people from other cultures. However, despite these benefits only rarely do study-abroad programs have CCC courses. Typically, visiting students learn about cultural differences in communication and collaboration through seminars that occur prior to their visits (Schubert and Jacobitz 2013; Parkinson 2007).

The purpose of this paper is, therefore, (1) to present a CCC skill-training curriculum that was developed and implemented in the US for visiting Singaporean students, (2) to examine the benefits of this curriculum for the students, and (3) to present lessons learned from implementing the course. 
The curriculum was designed using Daniel Goleman's emotional intelligence framework (Goleman 2004). Course benefits were assessed in two ways: (1) through pre- and post-course surveys asking students what they wanted to improve and what they learned as a result of the course and (2) through weekly student journal entries, which captured students' ideas, impressions, and activities while immersed in the class.

\section{Context}

\subsection{Course Overview}

A northeastern university offered an exploratory CCC course to visiting Singaporean students and host students in January 2015, as part of a month-long international winter exchange program with a university in Singapore.

The course, titled "Cross-Culture Collaboration: Theory and Practice" (CCCTP), consisted of two 90-minute classes per week over a period of three weeks. The course objectives were (1) to increase students' awareness of their personal and cultural viewpoints and habits and their differences from and similarities to their peers; (2) to provide students with communication tools that take account of these awarenesses; and (3) to give students opportunities to practice intercultural communication in an environment where it is safe to make mistakes, to experiment with new ideas, to ask sensitive questions, and to learn from each other.

The instructional team for the course consisted of a US-born lecturer from the host university's writing program; a Korean postdoctoral researcher in Engineering Education; a South Indian and a Ghanaian teaching assistant (TA); a German guest lecturer who had directed intercultural programs worldwide; and a US-born host university residential life advisor who lived with the Singaporean students in their dormitory.

During the course, the instructional team introduced CCC skills, including communicating effectively with people from different countries; recognizing and overcoming sources of tension arising from another's background; identifying the strengths and weaknesses of team members from different cultures; and identifying, monitoring, and seeking support for students' chosen areas of growth.

Forty students from a university in Singapore and nine from the host university took the CCCTP course. The course was a requirement for the visiting students, who were all also registered in a variety of technical classes where they participated in team projects with the host students. The course was no-credit for the visiting students and pass/fail for host students.

\subsection{Emotional Intelligence Framework}

The fundamental premise of the CCCTP course was that significant improvement in students' ability to collaborate effectively depends not only on cognitive learning of relevant concepts but also on experiential learning - that is, learning to consider one's capacity for bias and to explore new ways to communicate with and respond to others. Therefore, the main objectives of CCCTP were designed to build a foundation of emotional intelligence in students.

Emotional intelligence is the ability to recognize, understand, and manage ones' own and others' emotions. People with high emotional intelligence are measurably confident, good at working toward goals, adaptable, and flexible. Teaching emotional intelligence provides an approach to experiential learning that is easily understood by students, supported by years of research, credibly critiqued, and adaptable to a variety of learning situations. Introduced by psychologists Peter Salovey at Yale and John Mayer at the University of New Hampshire in 1990 (Salovey and Mayer 1990) and further developed and popularized by Daniel Goleman (Goleman 1995), the concept of emotional intelligence has proved to be a flexible and resilient tool with which to provoke and guide behavior change.

The instructional team used the emotional intelligence framework, as defined by Goleman (2004), to provide students with a structured way of understanding their feelings and behaviors in collaborative settings and also as a set of concepts against which students could measure their learning. To model the concepts of emotional intelligence for the class, the instructional team worked collaboratively during class sessions. The instructors considered themselves full participants in the class along with the students, with perhaps the addition of a few years' more experience with intercultural communication.

\section{Course Lesson Plan and Topic}

This section of the paper presents the CCCTP course lesson plan. We also include the rationale for each lesson plan activity along with its connections to the emotional intelligence framework.

\subsection{Session 1: Building Trust, Forming Teams, and Setting Expectations}

The overarching premise of the CCCTP course is that effective collaboration is built on trust among people who differ in a variety of ways. To help the students learn to build trust while collaborating, trust must be built in the 
class - trust between students and instructional team, and trust among the students themselves. Therefore, the first class session was built around forming trusting relationships grounded in shared vulnerability and managed risk.

\subsubsection{Nametags, Trust, and Metacommunication}

The first exercise of the class began just after the students picked up their blank nametags. They were asked to take two nametags and to write their name on one and draw a picture that somehow represented them on the other. Once the students were seated, the formal part of class began with a discussion, carried on lightly and with humor, of the experience of deciding on and then drawing a picture on their nametags. The discussion opened with the question, "What happened to you when you heard that instruction? What was your reaction to it?" Student reactions generally fell into four categories. Some were excited: "Oh boy! I get to draw a picture!" Some were anxious: "Oh, no! I have to draw a picture!" Some were annoyed: "Oh, no... it's going to be one of those kinds of classes...." Some had no particular response at all. Four main lessons were drawn from this discussion, all of them foundational to the class:

- We often have instantaneous, automatic reactions to events. We do not choose or control them; they simply arise.

- Our particular reactions are shared by others.

- All of our reactions are valid and understandable. There is no "right" reaction, although different reactions may have different effects.

- It is interesting, and perhaps a relief, to acknowledge, identify, and talk about these reactions - their automaticity and their specificity—without judgment.

We named these automatic emotional responses "uh-oh moments," in recognition of the fact that two of the response categories, the anxious and the annoyed, often cause mischief in collaborations, the one being tinged with fear and the other being tinged with anger. It is vital to call attention to, name, and discuss these automatic reactions because when they go unnoticed or unmentioned, they can unwittingly become the basis of attitudes and behaviors that inhibit effective collaboration. Being able to recognize and talk about their reactions was an important step in building emotional intelligence for the class. As the class progressed, noticing the occurrence of "uh-oh" moments during the class became an ongoing thread, begun by the instructors but soon taken up by the students.

Having noted the kinds of responses evoked by the request to draw self-representing pictures, we turned to a second part of the nametag discussion, the content of the pictures. The pictures were wildly diverse. Some were silly, others were astonishingly creative, and still others were eloquent, crude, haphazard, or profound. All of the instructors and some of the students shared their pictures with the whole class, discussing them with wit, self-deprecating humor, and thoughtful appreciation.

Sharing the pictures formed a second element of our emerging foundation of trust. The experience demonstrated that although we are all very different in temperament and talent, each of us has something valuable to contribute to our joint endeavor, and each of our various experiences is valid and worthy of time and respect.

The nametag discussion was followed by discussions about common experiences with collaboration, both positive and negative; student expectations and motivations; and the class structure. Instructors and TAs elicited commonalities and differences, commenting on our shared - if sometimes shrouded - humanity, and in particular on our shared vulnerabilities. We shared our own stories about difficult team experiences and how our automatic reactions to people and situations hampered our productivity, in order to (1) model moderate risk-taking, (2) establish common ground with the students, and (3) demonstrate the value and the need for honest communication and the mutual learning that results from it.

The underlying goal of this opening discussion was to begin creating an environment in which the students would feel able to express their differences and their vulnerabilities. A crucial factor in reaching this goal was the way that we modeled of such sharing.

\subsubsection{Communication and the Johari Window}

We wanted the students to engage with each other during the class. In particular, we wanted the visiting and the host students to work together on a collaboration-focused task so that each group could learn about the other's culture. Therefore, we assigned the students to teams and gave them the task of building websites on which they could freely record what they learned and experienced about collaboration. The task was intended to challenge the teams to operate with both individual responsibility and mutual support.

Our criteria for setting up the teams were straightforward: Each team was to include four students, at least one of 
them from the host university student and at least one of them a person with expertise in building a website. The website assignment was designed to engage the students in questions of self-awareness, self-regulation, and social skills as they planned and executed the decisions that would result in a collaborative project.

In addition, students were asked to submit individual weekly journal entries. An instructor read these entries, provided suggestions based on them, and used them to assess students' development of CCC skills. The journals were either on paper or electronic, and students could freely write in them about what they had learned or realized about themselves. Guiding questions were provided to help students think about their entries, such as: What did you notice about a collaborative situation? What did you wonder about? What caused you discomfort? What risk(s) did you take? What did you learn about yourself and/or others? How did you recover from mistakes?

The formation of the website teams was followed by a discussion that gave the students some cognitive structure to help them think about forming relationships and teams. Two principles from communications theory were explained: (1) We are always communicating, and (2) every communication has both an element of information and an element of emotion, though in widely varying proportions (Watzlawick et al. 2011). As we explained these principles, we also modeled them for the class by pointing out examples of the principles lightly, in a way that modeled openness. For example, while explaining the first concept, the students were asked, "What are our TAs communicating right now?" This question had the potential to be somewhat embarrassing for the TAs, who weren't expecting it. But the TAs responded with good humor and a bit of self-deprecation, as if to say, "We're not always paying $100 \%$ attention either!" Such modeling with humor was intended to make it safer for the students to relax, be themselves, and be less than perfect.

Finally, we presented a communication tool called the "Johari window." The Johari window is a framework that divides information about an individual into four quadrants: public (known to self and others), private (known to self/unknown to others), blind (known to others/unknown to self), and unknown (Ingham and Luft 1955). We used this framework to talk about what information had already, in the course of the day's class, shifted from one quadrant to another, and what had happened as a result. We identified the emergence of trust and openness and dissected their component parts as a way of demonstrating that trust and openness are not accidental but rather can often be proactively built. We also described possible experiences of mistrust or discomfort that participants might have experienced during the class and mapped these responses onto the Johari window in order to model and validate a wider range of potential responses than the students might have been comfortable revealing.

\subsection{Session 2: Risks and Benefits of Exploring Another's Worldview}

The second session of the CCCTP course focused on issues of culture. The host university's Director of International Affairs delivered the class lecture.

The session had three main components. First, the guest speaker provided several definitions of culture, a well-known system of value dimensions developed by Geert Hofstede, several analyses of cultural differences in nonverbal behaviors, and a case study comparing German and American industrial planning methods.

Second, the class included a discussion period during which students in groups of three compared their experiences of cultural differences with regard to punctuality and interrupting. Despite the fact that most of the students were from Singapore, the discussions were revealing. The Singaporean students were surprised to discover that they experienced nuanced differences even within their presumably homogeneous culture. The host students, among whom were some international students, contributed their divergent viewpoints.

Third, the class participated in a discussion about the discussion: a meta-conversation about their experiences of interrupting and being interrupted during the course of the preceding discussion, the "uh-oh" moments of automatic reaction they had felt, and their responses to those experiences. We challenged the students to try a behavior that went counter to their culture, to experiment and see what the experience felt like in this safe environment, and we discussed the difficulties that come with changing behavior and assessing its different effects.

The aspects of emotional intelligence targeted in this class were self-awareness, empathy, and social skills. The students gained information about their biases and preconceptions from the lecture and experienced those differences, and to some extent the power of those differences, in their discussions. Being able to participate in discussions about personal and cultural differences, moderated by the instructional team, was a new experience for some students, who commented on their surprise at the unexamined assumptions uncovered during the class.

Beginning with this class session, reference to cultural differences became a recurrent topic of discussion in the class. Both visiting and host students began to record in their journals what they thought might be evidence of cultural differences in the form of observations of themselves, their colleagues in the class, and others whom they 
encountered around the campus and in off-campus trips.

\subsection{Session 3: Interrupting Reactions to Find More Effective Responses}

The third class session covered content essential to moving the students from heightened self-awareness to an improved capacity for self-regulation. We informed the students that one key to improved collaboration was an improved ability to notice one's "uh-oh" moments and then to notice when an emotional reaction does not serve one's purpose.

\subsubsection{Identifying and Interrupting Suboptimal Reactions}

The reactions the students had in the first class to the "draw a picture" exercise were recalled, specifically their automaticity and, for some students, their attendant feelings of discomfort. We shared numerous examples to demonstrate how common such reactions are and how frequently and unpredictably they are triggered. We examined the various ways in which impulsive reactions might interfere with the kinds of relationships on which effective collaboration depends. These reactions - for example, frustration, annoyance, inauthentic cheeriness, fear, threat, overzealousness, anger - can underlie such detrimental behaviors as defensiveness, withdrawal, aggression, and stereotyping. They can actively disrupt or passively inhibit communication, creativity, and open engagement.

We then discussed, largely in the form of personal stories, the causes of these reactions. Reactions may be triggered, for example, by miscommunication or misinterpretation rooted in differences of culture or personality, differences in goals or values, environmental conditions such as resource or time constraints, or the state of formation of a team. Some of these triggers can be managed, and others cannot. Known cultural differences, for example, can be discussed, but many cultural differences may not be explicitly recognized. Paying attention to what these impulses feel like and understanding what provokes them can help us regulate ourselves when they occur.

\subsubsection{Tools for Improving Collaborative Behavior}

During the second part of the session, we considered what we might do, individually and as collaborators, to build emotional intelligence and mitigate the impact of our emotional reactions on others and ourselves.

We presented the students with a set of communication tools in the form of Bingo cards. We told the students that the cards listed tools for setting a team back on track, most of which they already knew, and that there is no one right way to re-engage a team. Rather, effective team members try a strategy, observe whether it helps, and if it doesn't they try another. The need for patience and a trial and error approach was illustrated with several stories, such as one about complex international negotiations that have taken years to succeed, encountering many dead ends and false starts along the way.

The tool of "framing"- making one's intention or concern explicit as a metacommunication, in advance of offering one's point - was singled out as useful in cross-cultural teams, where unpredictable misunderstandings are likely. The students began to use framing, and to point out uses of it, almost immediately in class discussions. Introductory phrases such as, "I don't mean to cause offense, but I want to make sure I understand you," and, "This may seem obvious, but...," began to be routinely heard in the classroom, and the students reported their value in other situations as well.

\subsubsection{The Five Conversations of Collaboration}

In addition to the Bingo card of tools, we presented the students with a conceptual framework for thinking about collaboration as a set of five layered conversations about tasks, goals, process, relationships, and values, each of which needs to be initiated in the course of an effective collaboration. Using this framework, one strategy for improving a team's effectiveness is to consider changing the level of conversation. For example, if a team becomes enmeshed in accomplishing tasks, its attention can be turned to process (how will we decide our priorities?), or to goals (Are we accomplishing the right tasks?), or to relationships (Are people being heard and appreciated as they complete these tasks?).

\subsubsection{Using the Tools in a Website Project}

For the rest of the class, the students met in their teams to plan their website projects and to begin putting the tools and strategies into practice. In these meetings, the students would begin to build on the self-awareness and self-regulation we had been examining and to experiment with new social skills, in particular managing relationships, looking for opportunities to learn from and support each other, and using persuasion and feedback to build their sense of the team. We anticipated that this learning would show up in their ability to offer feedback to each other, to persuade and be persuaded, to keep their teams focused, to make good decisions, and to sort out conflict. 


\subsection{Session 4: Exploring Underlying Values - Questions, Feedback, and Appreciation}

The fourth session opened with a debrief of the teams' website design meetings: what they observed about their collaboration, what tools and concepts they used, what awarenesses they noticed, and what challenges they faced. The goal of this discussion was to have the students learn from each other and appreciate their collective ability to experiment and problem-solve by using and sharing what they were learning. As the teams shared their experiences, the lesson was vividly brought home that no two teams work in the same way, learn the same lessons, or face their challenges with the same strategies. Insights sparked other insights, and even within a team the students realized that they had learned from their experiences in a wide variety of ways.

The rest of the class was taken up with three exercises, done in groups of three students each, with the instructional team moving around the room to guide the discussions.

\subsubsection{Values Underlie Complaints: Using a Question Protocol to Illuminate Problems}

The first exercise used a series of structured questions to demonstrate the value of gently probing complaints in order to uncover the underlying values that complaints often mask. The students experienced how thoughtful questioning can lead to deeper understanding of broad issues and root causes, thereby building trust and resulting in more lasting problem-solving. They observed that we often mean more than we are aware of. Many of the students discovered, either directly or by observation, that while the act of questioning can be uncomfortable, the mutual value of what is learned can offset the discomfort.

This exercise was designed to teach empathy, specifically the ability to understand variations in language and in the emotional makeup of other people. The students were explicitly coached to watch for and ask about cultural differences, to use the questions to avoid preconceptions, to seek out and learn from others' points of view, to assess and adjust to others' emotional states, and to inquire into what underlies another's complaint.

\subsubsection{Listening in a Way That Enhances the Value of Feedback}

The second exercise asked the students to give each other feedback on some aspect of their participation that they might improve. First, we discussed how a listener's attitude affects the value of the feedback that is offered. Then, we discussed the relationship between effective feedback and underlying values. A structured formula was offered for practice, leading to a discussion comparing the value of conversational templates for practice with the importance of authenticity in real interactions. Learning objectives included several experiences: that feedback can be delivered more easily when it is phrased responsibly ("I" statements), that feedback may say more about the giver than the receiver ("my perception is"), that an exchange of feedback can reveal important underlying values, and that using feedback well requires collaboration and trust.

This exercise was aimed at building the students' confidence in their ability to manage their relationships with others. Specifically, the goals were to learn about offering feedback in a useful way, to gain more options for action when a teammate is not being productive, to build trust through honest communication, to recover from mistakes by apologizing effectively, and to encourage professional behavior.

\subsubsection{Sharing Appreciation}

In the final exercise of the day, each student was asked simply to tell the other two students in their group something they genuinely appreciated about them. This exercise is linked to the motivation component of emotional intelligence, the "passion to work for reasons that go beyond money or status; a propensity to pursue goals with energy and persistence" (Goleman 2004). An exchange of authentic appreciation led a number of students to explore the value of appreciation, as well as what drives them to achieve and what they think about others' motivations.

\subsection{Session 5: Practicing Skills and Concepts in Team Meetings}

The fifth session consisted of a debrief of what the students had been noticing and working on in their various collaborative activities, followed by a work session for teams to meet and work on their websites.

As part of the debrief, we discussed the differences between experiential learning and conceptual learning. We discussed the ways in which learning about communication and behavior is different than learning information, in that it is not sequential, is very personal, and is highly variable. Often, learning continues well after a class ends; often it happens by surprise. A great deal of experiential learning happens from listening to others share their insights and during opportunities to practice in an environment where humor is cultivated and mistakes are encouraged. One intention of this discussion was to prepare them for the continued unfolding of realizations out into the future.

Following a rich and wide-ranging discussion, we had the students review and assess their goals for the class, firm up their plans for completing their websites, and decide how to present their websites to the class in our next meeting. 


\subsection{Session 6: Celebrating Our Learning and Looking Ahead}

Our sixth and last session was given over to two activities. First, the two TAs presented a summary of two articles about team learning and intelligence. The first article, by Woolley and colleagues (Woolley et al. 2010), analyzed data from over 600 people who worked in small teams to solve a variety of tasks. The second article, by Druskat and Wolff (2001), described ways in which emotional intelligence has been built into the culture of successful companies. The purposes of this discussion were to validate what the students had been learning and experiencing with some published data, to expand their thinking about future collaborative endeavors, and to give the TAs an opportunity to teach.

Next, the teams presented their websites and explained what they had learned and experienced about collaboration in the process of developing them during the three weeks of the class. Each team demonstrated a rich combination of creativity, insight, dedication to its relationships, and significant learning. The presentations themselves, while not necessarily polished, were expressive and lively. Each conveyed a strong sense of camaraderie, and each had a distinctive team personality, ranging from exuberant to whimsical to earnest and thoughtful.

\section{Method - Examination of Course Benefits}

This section of the paper highlights the benefits of the CCCTP course on visiting and host university students. The course benefits were investigated from the results of a 40-item survey, which was administered before and after the course, and entries from weekly student journal entries.

The 40-item survey was originally developed for team-based courses at the host university but was modified to assess students' perceptions of their abilities with regard to emotional intelligence (EI) and cross-culture collaboration. Specifically, the survey assessed students' beliefs on (1) the need to improve a range of skills associated with five EI factors before they had taken the course (pre-survey) and (2) the skills that they learned from the course (post-survey). Students completed the survey online prior to the first class, rating each item according to the following scale: " $1=$ I'm okay with this," " $2=$ I wonder whether I need to work on this," " $3=$ I need to work on this," and " $4=\mathrm{I}$ really need to work on this!" On the last day of the class, students re-took the same survey (post-survey) but were asked to rate the items using a different scale: " $1=$ This item didn't apply to me," " $2=$ I wish I had learned more about this," " $3=\mathrm{I}$ learned about this," and " $4=\mathrm{I}$ learned something valuable about this." Prior to administering the survey to students, each survey item was validated. The members of the instructional team and an engineering faculty member reviewed the items for clarity and checked whether they adequately represented the factors.

\section{Results}

\subsection{Survey Results: Students' Perceived Learning on Five Emotional Intelligence Factors}

Table 1 shows the results from 41 of the 49 students who completed the pre- and post- survey. The pre column shows the percentage of students who answered, "I need to work on this" or "I really need to work on this!" prior to the course. The post column shows the percentage of students who answered, "I learned about this" or "I learned something valuable about this" after the course. Cronbach's alphas, which measures how closely related the items belonging to each factor are, were determined using students' pre- and post-survey scores. Lastly, each factor's mean was determined by averaging the percentages for the items belonging to each factor.

Table 1. Pre- and post- survey measures of students' perceived learning of emotional intelligence factors

\begin{tabular}{llll}
\hline Self-Awareness Factor & $\begin{array}{l}\text { Pre } \\
(\boldsymbol{\alpha}=\mathbf{0 . 7 9 )}\end{array}$ & $\begin{array}{l}\text { Post } \\
(\boldsymbol{\alpha}=\mathbf{0 . 7 1 )}\end{array}$ & Difference \\
\hline 1. Be sure of what is expected of me & $24 \%$ & $88 \%$ & $64 \%$ \\
2. Get my point across & $39 \%$ & $88 \%$ & $49 \%$ \\
3. Be open to others' ideas & $17 \%$ & $85 \%$ & $68 \%$ \\
4. Look for the value in criticism even when I disagree & $49 \%$ & $83 \%$ & $34 \%$ \\
5. Voice my ideas in a way that helps move things forward & $63 \%$ & $76 \%$ & $13 \%$ \\
6. Keep things moving efficiently, in meetings and at work & $37 \%$ & $73 \%$ & $36 \%$ \\
7. Feel more satisfied with my work & $32 \%$ & $71 \%$ & $39 \%$ \\
Self-Awareness factor mean & $37 \%$ & $80 \%$ & $43 \%$ \\
\hline
\end{tabular}




\begin{tabular}{|c|c|c|c|}
\hline Self-Regulation Factor & $\begin{array}{l}\text { Pre } \\
(\alpha=0.84)\end{array}$ & $\begin{array}{l}\text { Post } \\
(\alpha=0.81)\end{array}$ & Difference \\
\hline 1. Listen to others constructively, even when I disagree & $27 \%$ & $93 \%$ & $66 \%$ \\
\hline 2. Listen carefully and ask questions to make sure I understand & $20 \%$ & $93 \%$ & $73 \%$ \\
\hline 3. Accept feedback well & $29 \%$ & $90 \%$ & $61 \%$ \\
\hline $\begin{array}{l}\text { 4. Speak in a way that helps resolve conflict, rather than being angry, } \\
\text { withdrawn, or resistant }\end{array}$ & $44 \%$ & $88 \%$ & $44 \%$ \\
\hline 5. Know when to listen and when to speak & $29 \%$ & $78 \%$ & $49 \%$ \\
\hline 6. Behave professionally & $27 \%$ & $71 \%$ & $44 \%$ \\
\hline 7. Behave respectfully towards others, whether or not they are present & $20 \%$ & $71 \%$ & $51 \%$ \\
\hline $\begin{array}{l}\text { 8. Know when to say what I think and when to keep my views to } \\
\text { myself }\end{array}$ & $59 \%$ & $68 \%$ & $9 \%$ \\
\hline 9. Be more patient & $27 \%$ & $63 \%$ & $36 \%$ \\
\hline Self-Regulation factor mean & $31 \%$ & $79 \%$ & $48 \%$ \\
\hline Motivation Factor & $\begin{array}{l}\text { Pre } \\
(\alpha=0.73)\end{array}$ & $\begin{array}{l}\text { Post } \\
(\alpha=0.75)\end{array}$ & Difference \\
\hline 1. Find out what my teammates need & $68 \%$ & $93 \%$ & $25 \%$ \\
\hline 2. Make sure people are appreciated for good work & $34 \%$ & $83 \%$ & $49 \%$ \\
\hline $\begin{array}{l}\text { 3. Make sure roles and responsibilities are both clearly defined and } \\
\text { wisely assigned }\end{array}$ & $34 \%$ & $78 \%$ & $44 \%$ \\
\hline 4. Do my best work regardless of how the rest of my team is doing & $29 \%$ & $73 \%$ & $44 \%$ \\
\hline 5. Help other people to do their jobs & $17 \%$ & $68 \%$ & $51 \%$ \\
\hline 6. Motivate my teammates to do their best & $61 \%$ & $63 \%$ & $2 \%$ \\
\hline Motivation factor mean & $41 \%$ & $76 \%$ & $35 \%$ \\
\hline Empathy Factor & $\begin{array}{l}\text { Pre } \\
(\alpha=0.76)\end{array}$ & $\begin{array}{l}\text { Post } \\
(\alpha=0.37)\end{array}$ & Difference \\
\hline $\begin{array}{l}\text { 1. Recognize when people behave differently than I do because of } \\
\text { culture }\end{array}$ & $39 \%$ & $93 \%$ & $54 \%$ \\
\hline 2. When teammates complain, find out what they really want & $56 \%$ & $93 \%$ & $37 \%$ \\
\hline 3. Avoid preconceptions by asking lots of questions & $51 \%$ & $88 \%$ & $37 \%$ \\
\hline 4. Seek out and learn from others' points of view & $44 \%$ & $88 \%$ & $44 \%$ \\
\hline 5. Read and adjust to the feelings/emotional states of teammates & $46 \%$ & $80 \%$ & $34 \%$ \\
\hline Empathy factor mean & $47 \%$ & $88 \%$ & $41 \%$ \\
\hline Social Skills Factor & $\begin{array}{l}\text { Pre } \\
(\alpha=0.89)\end{array}$ & $\begin{array}{l}\text { Post } \\
(\alpha=0.75)\end{array}$ & Difference \\
\hline 1. Offer feedback to others in a useful way & $56 \%$ & $98 \%$ & $42 \%$ \\
\hline 2. Understand how teams build trust & $68 \%$ & $98 \%$ & $30 \%$ \\
\hline 3. Give constructive feedback to teammates & $29 \%$ & $93 \%$ & $64 \%$ \\
\hline $\begin{array}{l}\text { 4. Collaborate actively with teammates through frequent } \\
\text { communication }\end{array}$ & $32 \%$ & $85 \%$ & $53 \%$ \\
\hline $\begin{array}{l}\text { 5. Make and manage effective agreements about how the team should } \\
\text { work together }\end{array}$ & $37 \%$ & $78 \%$ & $41 \%$ \\
\hline 6. Help my team make good decisions & $46 \%$ & $76 \%$ & $30 \%$ \\
\hline 7. Help the team make effective decisions that everyone supports & $51 \%$ & $76 \%$ & $25 \%$ \\
\hline 8. Help my teammates sort out conflicts & $59 \%$ & $73 \%$ & $14 \%$ \\
\hline 9. Know what to do when a teammate is not doing their job on time & $73 \%$ & $73 \%$ & $0 \%$ \\
\hline
\end{tabular}


10. Help my team stay focused

11. Encourage my teammates to behave professionally

12. Help teammates with differences get along

13. Know how to persuade others

Social Skills factor mean

$\begin{array}{lll}49 \% & 71 \% & 22 \% \\ 41 \% & 68 \% & 27 \% \\ 56 \% & 66 \% & 10 \% \\ 71 \% & 61 \% & -10 \% \\ 51 \% & 78 \% & 27 \%\end{array}$

The Pre column shows that in the beginning of the course, less than half of the students believed they needed to work on the skills associated with the Self-Awareness, Self-Regulation, Motivation, and Empathy factors. After the course, however, students believed they had learned something valuable across all five EI factors. Seventy-six percent or more of the students believed that they learned about five EI factors. This result shows that students learned a great deal about certain aspects of EI and CCC skills that in the beginning of the course they had not considered to be something they needed to learn. Table 1 also shows the Cronbach's alpha for each factor. Each factor has Cronbach's alpha greater than or equal to 0.71 (except for post-score Empathy factor), indicating adequate internal consistency.

\subsection{Survey Results: Differences between Host and Visiting University Students}

To determine whether there are differences between how visiting and host students perceived their learning experiences on five EI factors, independent sample $t$-tests were performed. Using the sum of the item scores from each factor, the $t$-tests revealed no significant difference between host and visiting students' perceived learning differences on abilities associated with Self-Awareness, Motivation, Empathy, and Social Skill factors (Table 2). This result suggests that the students benefitted from the course independent of their experience.

The only significant difference in learning experience between the two groups of students was on the Self-Regulation factor. The visiting students had a higher score for this factor $(M=27.39, S D=4.04)$ than the host students $(M=$ $23.75, S D=6.23)$. The result was associated with a statistically significant effect, $t(39)=2.05, p=0.047$. When further investigated to determine which items within the Self-Regulation factor showed large mean differences (greater than .5) between the two groups of students, the following items were identified: "Listen to others constructively, even when I disagree"; "Know when to listen and when to speak"; and "Behave respectfully towards others, whether or not they are present."

Table 2. Comparison of visiting and host students' emotional intelligence factor mean scores after CCCTP course

\begin{tabular}{|c|c|c|c|c|c|c|}
\hline Factor (\# of items) & $\begin{array}{l}\text { Visiting }(N=33) \\
\text { Host }(N=8)\end{array}$ & $M(S D)$ & $t$ & $d f$ & $\begin{array}{l}\text { Sig. } \\
\text { (2-tailed) }\end{array}$ & Cohen's $d$ \\
\hline \multirow{2}{*}{ 1. Self-Awareness (7) } & Visit & $21.18(3.16)$ & \multirow{2}{*}{1.29} & \multirow{2}{*}{39} & \multirow{2}{*}{0.204} & \\
\hline & Host & $19.63(2.56)$ & & & & \\
\hline \multirow{2}{*}{ 2. Self-Regulation (9) } & Visit & $27.39(4.04)$ & \multirow{2}{*}{2.05} & \multirow{2}{*}{39} & \multirow{2}{*}{0.047} & \multirow{2}{*}{0.81} \\
\hline & Host & $23.75(6.23)$ & & & & \\
\hline \multirow{2}{*}{ 3. Motivation (6) } & Visit & $17.70(2.82)$ & \multirow{2}{*}{1.37} & \multirow{2}{*}{39} & \multirow{2}{*}{0.178} & \\
\hline & Host & $16.13(3.27)$ & & & & \\
\hline \multirow{2}{*}{ 4. Empathy (5) } & Visit & $16.24(1.90)$ & \multirow{2}{*}{-0.51} & \multirow{2}{*}{39} & \multirow{2}{*}{0.616} & \\
\hline & Host & $16.63(2.00)$ & & & & \\
\hline \multirow{2}{*}{ 5. Social Skills (13) } & Visit & $39.30(4.91)$ & \multirow{2}{*}{0.57} & \multirow{2}{*}{39} & \multirow{2}{*}{0.572} & \\
\hline & Host & $38.25(3.49)$ & & & & \\
\hline
\end{tabular}

\subsection{Student Journal Excerpts}

Student reflections varied greatly because students were given free rein to express themselves, but they all contained a range of evidence that students found the class valuable. Sample journal excerpts are presented in Table 3. 
Table 3. Sample CCCTP course student journal excerpts

When meeting new people, though we need to be cautious given the situation, it is also rewarding to be open-minded and willing to share experiences. It is only by such measures that we can truly collaborate across cultures.

... as I break down what I need to work on, I realize how it is very important, but also very doable. Even right now, as I explain the notecard, it makes me realize how very important it is to be a more patient, better-listening group member.

One of the biggest things I took away from this class, when the topics of vulnerability, confidence, and communication [were] discussed, was that technical skills can only get you so far. I really need to work on my people skills.

It was an eye-opening experience because it challenged a lot of my viewpoints.

Due to these two interactions with the Singaporeans, I've decided to speak more. I want to be there for people and to not be silent, but to listen and to ask the right questions. I want to be a better communicator.

I confess that I prefer to blame people for the choices they make when it comes to they work. Perhaps that may still hold true for some people, but I guess it is not a trivial matter... Thus, I am actively thinking how I can help people work well together despite the cultural norms.

One great takeaway from this week's lessons were about courage in communication. I think courage is a necessary ingredient in developing relationships. One should not be afraid to fail when trying to know more about the other person. There may be times where it becomes awkward, embarrassing or conflicting, but I trust that these are links that connect the dots together in a relationship. It's not going to be easy, but it's worth [it] to see how relationships between people can blossom.

I guess I notice how culture really seeps into almost every facet of how things are done in different places. Though it can be a source of immense frustration, I thoroughly love how different we are, and how human we are.

The excerpts in Table 3 show the kinds of important messages students took from the course. In many cases, students said that they learned to apply familiar communication tools to new kinds of situations, because they had expanded their self-confidence and their ability to question and to empathize.

\section{Discussion and Lessons Learned}

For most people, learning to communicate and collaborate across culture happens through experience, practice, and mentorship. To facilitate students' development in communication and collaboration, we created an experiential learning environment, developed strong relationships with students, and modeled the behaviors and attitudes that we were promoting. We believe these were essential elements for the success of the course.

\subsection{Experiential Learning}

Experiential learning was embedded in the course. In class, students had the opportunity to think about, experiment with, and practice ways to work together with peers from different countries.

Writing assignments allowed students to share their observations, explorations, achievements, and challenges when communicating and collaborating with others. In these assignments, students (1) recorded the impact of concepts and tools taught in class on their lives; (2) learned to observe themselves and others, identifying cues and signals that carry meaning and approaching that meaning with curiosity; (3) wrote about experiences/trials as discovery or adventure; and (5) received feedback and suggestions from instructors.

Technical (or other subject) classes that the students concurrently took with the CCCTP course served as laboratories in which to practice the tools and concepts that they had learned. Students used the tools and concepts differently depending on their needs, abilities, background, and circumstances. These classes gave students a wealth of successes, failures, and interesting stories to share with each other. The students said that they learned some of their most valuable lessons from each other.

\subsection{Role of the Instructional Team}

Another element leading to the success of the CCCTP course was the quality of the relationships between the students and the instructional team. Both the positive results of this class and the students' marked enthusiasm derive 
not only from the concepts taught but also from the authentic relationships students built with the instructional team and from observing how the team modeled emotional intelligence.

It was important for the instructional team to be themselves as emotionally vulnerable as they asked the students to be. If the team was to have success in asking students to be aware of and to share their moods, emotions, strengths, weaknesses, needs, and drives, the team needed to be similarly aware and willing to share. In other words, the instructional team needed to be emotionally intelligent: self-aware, able to share feelings and reactions in the moment, willing to make mistakes and look foolish, at ease with exploring along with students in a state of confident curiosity, and motivated to build lasting personal relationships with students.

\section{Conclusion}

We created a cross-cultural collaboration class to teach collaboration and communication skills to visiting and host university students. The CCCTP curriculum, which is based on Daniel Goleman's work with emotional intelligence, gave students a wealth of opportunities to learn, experiment, and share about communication and collaboration. Analysis of the pre- and post-surveys and of the journal entries indicates the value of our course for both student groups. We believe the course gave our students the tools, coaching, motivation, and tolerable failures that they needed to attain a much-expanded sense of what is possible in cross-cultural collaboration, as well as enough confidence and ideas to support continuing growth in collaboration skills.

\section{References}

Druskat, V. U., \& Wolff, S. B. (2001). Building the emotional intelligence of groups. Harvard Business Review, 79(3), 80-91.

Goleman, D. (1995). Emotional Intelligence. Bantam Books, New York: NY.

Goleman, D. (2004). What makes a leader? Harvard Business Review, 82(1), 82-91.

Ingham, H., \& Luft, J. (1955). The Johari window, a graphic model of interpersonal awareness. Western training laboratory in group development. UCLA Extension Office.

Parkinson, A. (2007). Engineering study abroad programs: formats, challenges, best practices. Online Journal for Global Engineering Education, 2(2), 2.

Salovey, P., \& Mayer, J. D. (1990). Emotional intelligence. Imagination, cognition and personality, 9(3), 185-211.

Schubert Jr, T. F., \& Jacobitz, F. G. (2013). Compact international experiences: Expanding student international awareness through short-term study abroad courses with substantial engineering technical content. Online Journal for Global Engineering Education, 7(1), 1.

Watzlawick, P., Bavelas, J. B., Jackson, D. D., \& O'Hanlon, B. (2011). Pragmatics of human communication: A study of interactional patterns, pathologies and paradoxes. WW Norton \& Company.

Wulf, W. (2004). An urgent need to change. The Bridge, National Academy of Engineering. 